\title{
Hybrid Damping in Higher Order Line Side Filter in Active Front-end Operation
}

\author{
Nilanjan Mukherjee and Vinod John
}

\begin{abstract}
Active front-end converter draws a special attention in drives application and distributed generation application where good power quality and robustness are essential. Damping is an essential part of design in LCL filter based grid-connected system from point of view closed loop stability. This paper presents a novel concept of state-space based hybrid-damping technique, which is an appropriate combination of passive and active damping, in order to make grid-interconnection reliable and robust which can ensure adequate stability margin in all possible grid interactive conditions.
\end{abstract}

Index Terms-Active front end converter (AFEC); hybrid damping; state-space based analysis; LCL filter.

\section{INTRODUCTION}

Active front end converters (AFEC) are becoming more and more popular nowadays in adjustable speed drives and distributed generation system because of its potential advantages of controlling line side power factor as well as output DC-link voltage [1]-[3]. On the other side, using this type of PWM converter can inject harmonic current to grid due to their PWM switching which essentially can disturb EMI sensitive loads in the grid side. As a solution, LCL filter can be well accepted [1]-[2] from point of view of meeting harmonic standards $[4,5]$ requirement, cost and fast dynamic performance especially in grid interactive cases. The main drawback of using this type of filter is resonance oscillation, which could affect system stability and at the same time, can badly affect the Total Demand Distortion (TDD) of line side current which is unacceptable. Damping of resonance in LCL filter is very essential in order to improve the quality (TDD) of the line side current and system stability. There are two distinct ways of controlling the resonance have been discussed in the literature in recent years like passive [6] and active damping [7]-[8]. Active damping is very useful for controlling such oscillation as it is based on modulation of the converter which essentially does not affect the system efficiency as well as can able to track shifting of resonance frequency. Passive damping is the most reliable and simplest way of controlling the resonance which can able to provide adequate damping even when converter is switched off. But

Manuscript received February 20, 2012; revised March 29, 2012. This work was supported in part of project by the NaMPET (National Mission on Power Electronics Technology), Govt. of India in Indian Institute of Science (IISc), Bangalore.

N. Mukherjee was with Power Electronics Group in Indian Institute of Science, Bangalore and is now with the Power Engineering and Power Electronics Group in Aston University, Birmingham, UK (e-mail: mukhern2@aston.ac.uk).

V. John is with Indian Institute of Science (IISc), Bangalore, India (e-mail: vjohn@ee.iisc.ernet.in). depending entirely on this type of damping not only affects the overall system efficiency but it also remains ineffective in case of shifting of resonance frequency due to grid impedance variation. In large inter-connected power system, having many power converters interconnected to same PCC, all the converters cannot be disconnected very frequently from the grid due to the limited operating life of breakers and contactors.

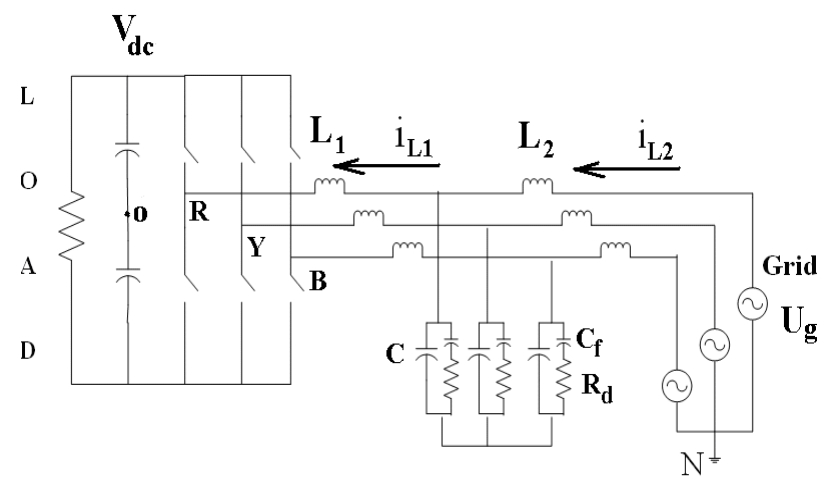

Fig. 1. Proposed Active front-end operation with suitable optimized R-C parallel damping.

In those kinds of situation, resonance oscillation could be easily propagated to/from the associated neighbouring system when main converter is switched off. In order to avoid this kind of unwanted phenomenon, combination of passive and active damping could be incorporated into the system to make grid interconnection much more reliable and robust. In this paper, concept of hybrid damping is proposed which uniquely combines the advantages of both passive damping and active damping at a time. The novel approach of ' $Q$ '-factor reduction has been adopted to validate the effectiveness of proposed hybrid damping. The 'Q'-factor has been reduced step by step with the help of suitable passive damping and then with the help of suitable closed-loop control algorithm super-imposing on that. The algorithm has been validated by using a 10KVA power PWM converter with LCL filter in $440 \mathrm{~V}, 50 \mathrm{~Hz}$ grid connected system under different practical conditions

\section{Suitable Passive DAMPING AND ANALYSIS OF DAMPING FACTOR}

The proposed power circuit consists of front-end converter with suitable passively damped LCL filter is shown in Fig. 1. Here, in this section, only the damping network as shown in Fig. 2, is considered to evaluate the damping performance. Passive damping directly affects the system efficiency. Hence, the passive damping network has to be properly chosen such that it does not reduce the efficiency too much. 
$\mathrm{R}-\mathrm{C}$ parallel damping network has been adopted in the paper Fig. 2 as this type of topology has some crucial potential advantages compared to simple capacitor-series-resistance damping topology [6] as that damping topology causes considerable loss especially in medium and high power level [6]. R-C parallel topology Fig. 2 consumes comparatively less overall losses (more efficient) due to bypassing capacitance ' $\mathrm{C}$ '. The R-C parallel network has been elegantly formed by just splitting the total capacitance $\mathrm{C}_{\mathrm{T}}$ of LCL-filter (Fig. 2) in into two parts like $\mathrm{C}$ and $\mathrm{C}_{\mathrm{f}}\left(\mathrm{C}_{\mathrm{T}}=\mathrm{C}+\mathrm{C}_{\mathrm{f}}\right)$ by some suitable factor ' $a$ ' $\left(\mathrm{C}_{\mathrm{f}} / \mathrm{C}=\mathrm{a}\right)$ and putting a resistance in series

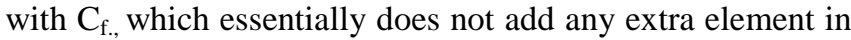
the circuit and at the same time, not increases the overall cost of the system compared to simple capacitor-series-resistance damping topology.

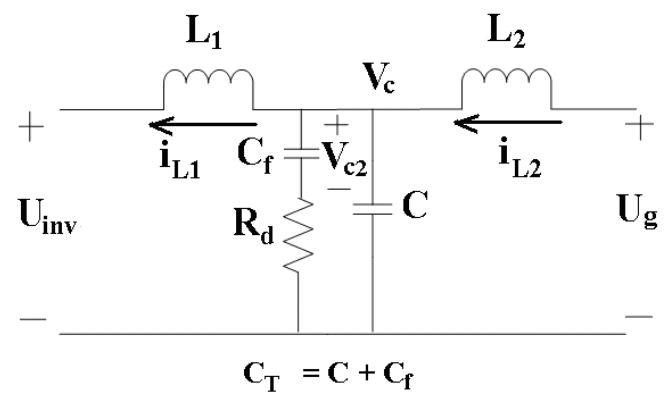

Fig. 2. Optimized R-C parallel damping network with LCL filter for AFEC

$V_{c}(s) / U_{i n v}(s)$ transfer function has been selected to calculate and compare damping performance in LCL filter. The 'Q'-factor could be easily calculated from the peak magnitude of the frequency response plot at resonance frequency. The $V_{c}(s) / U_{i n v}(s)$ transfer function is the only transfer function in LCL filter, which has a constant magnitude $(0 \mathrm{~dB})$ at the lower frequencies as can be seen in Fig 3. In this paper, $V_{c}(s) / U_{i n v}(s)$ bode plots (damped and un-damped) is drawn after normalization by factor 2 as $\mathrm{L}_{1}=$ $\mathrm{L}_{2}$ has been taken as design parameter and the resonance frequency has been selected as $\mathrm{f}_{0}=1 \mathrm{KHz}$ Fig. 3 The filter and damping network parameters are given in Table II.

This R-C parallel damping is not only dependent on $\mathrm{R}_{\mathrm{d}}$, but also on $C_{f} / C$ ratio [6]. Damping factor $(\xi \approx 1 / 2 \mathrm{Q})$ increases (or Q-factor reduces) for higher $C_{f} / C$ ratio and higher $\mathrm{R}_{\mathrm{d}}$ [6]. But, higher $C_{f} / C$ ratio or higher $\mathrm{R}_{\mathrm{d}}$ also leads to higher losses and reduces overall efficiency as shown in Fig. 5. The 'Q'-factor is calculated by using frequency plot of damped R-C circuit $V_{c}(s) / U_{i n v}(s)$ transfer function (1) by taking different ratios $\left(C_{f} / C\right)$ as shown in Fig. 4 , keeping $R_{d}$ to be fixed.

$\frac{V_{c}(s)}{U_{i n v}(s)}=\frac{L_{2}}{L_{1}+L_{2}} \frac{1+s C R_{d}}{s^{3}\left(L_{1} \| L_{2}\right) C C_{f} R_{d}+s^{2}\left(L_{1} \| L_{2}\right)\left(C+C_{f}\right)+s C_{f} R_{d}+1}$

It can be seen from Fig 4 that there is no such improvement of ' $Q$ '-factor after certain ' $\mathrm{a}$ ' $(=1)$ or $C_{f} / C$ ratio of 1 , for a given $R_{d}$. So, the factor ' $a$ ' can be suitably chosen as 1 . Thereafter, selection of $R_{d}$ has been done from loss analysis, at $50 \mathrm{~Hz}$ as well as switching frequency $(5 \mathrm{KHz})$ as shown in Fig. 5, keeping ' $a$ ' $=1$. It could be seen that, loss increases with increase of $R_{d}$, hence $R_{d}$ cannot be chosen too high but at the same time, it cannot also be chosen too low as damping performance would be affected. Around $R_{d}=25 \Omega$ could be engineering selection taking both points (damping and loss) into account

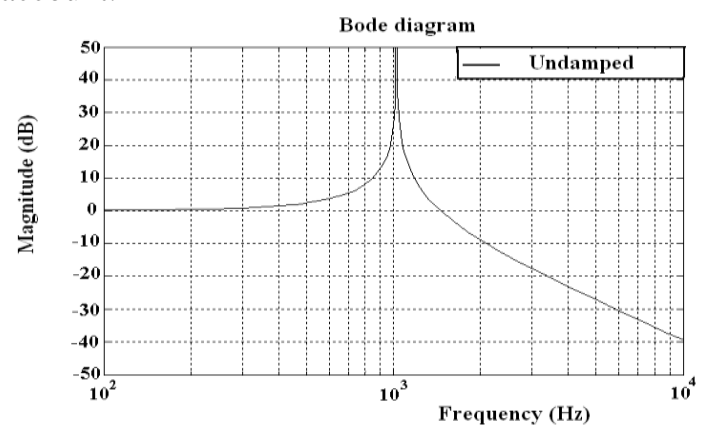

Fig. 3. Frequency response of capacitor voltage $\left(\mathrm{V}_{\mathrm{c}}\right) \mathrm{Vs}$ converter output $\left(\mathrm{U}_{\text {inv }}\right)$ transfer function in un-damped LCL filter (normalized by factor of 2).

\section{A. Reduction of ' $Q$ '-Factor and Shifting of Imaginary Pole-pair by Passive Damping}

The damping ratio $\xi$ is directly related to ' $Q$ '-factor or equivalently, $\xi \approx 1 / 2 \mathrm{Q}$. Reduction of ' $\mathrm{Q}$ '-factor increases the damping of resonance oscillation in the circuit. In other words, reduction of ' $Q$ ' indirectly means shifting of imaginary pole pairs more towards the left hand side of s-plane as shown in Fig. 6. The 'Q'-factor is obtained by calculating the peak magnitude of the normalized transfer function $V_{c}(s) / U_{i n v}(s)$ at resonant frequency. The ' $Q$ '-factor-reduction by means of frequency response plots are shown in Fig. 7 and Fig. 8 for different values of $R_{d}$.

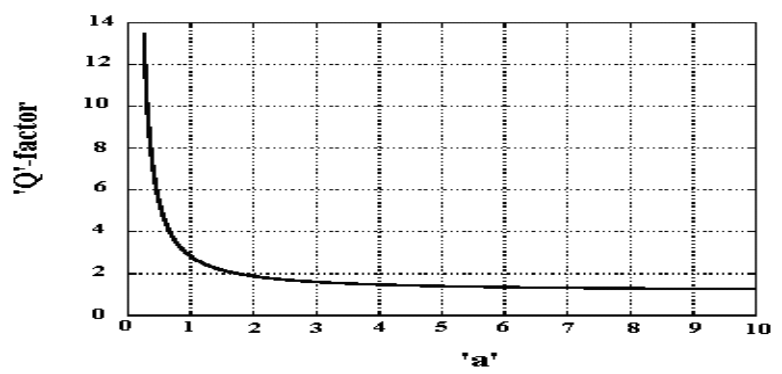

Fig. 4. Variation of ' $Q$ '-factor with $\mathrm{C}_{\mathrm{f}} / \mathrm{C}$ ratio, ' $\mathrm{a}$

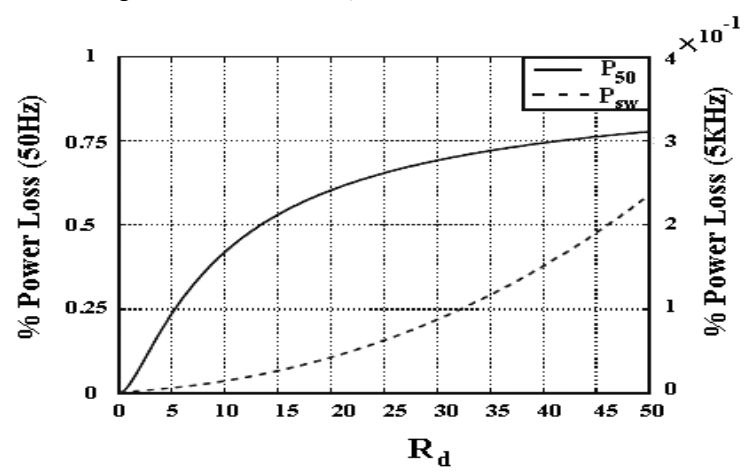

Fig. 5. Variation of losses in passively damped LCL filter with different $R_{d}$ 's (in $\Omega$ )

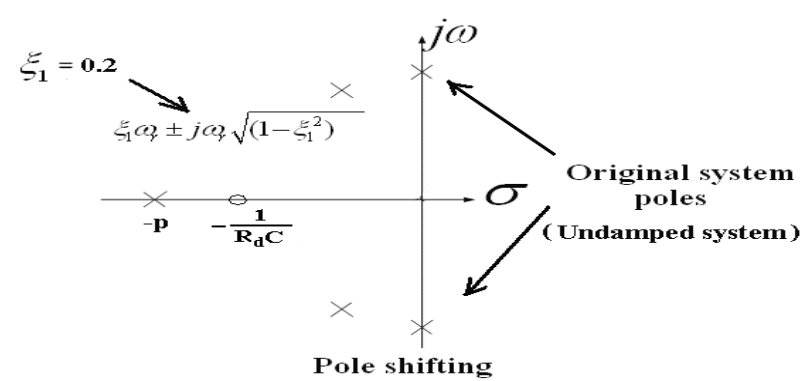

Fig. 6. Pole-zero plot of capacitor voltage $\left(V_{c}\right) V s$ converter output $\left(U_{i n v}\right)$ transfer function for passively damped and un-damped system. 


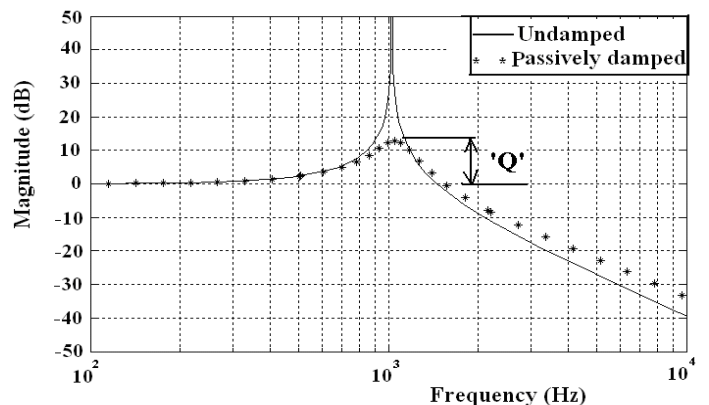

Fig. 8. Frequency response plot of capacitor voltage $\left(V_{c}\right) V s$ converter output $\left(U_{i n v}\right)$ transfer function in passively damped $\left(\mathrm{R}_{\mathrm{d}}=25 \Omega\right)$ and un-damped case (normalized by factor of ' 2 ').

The poles and zeros of the system with this passive damping can be mathematically expressed as following:

$\mathrm{s}=0,-\mathrm{p},-\xi_{1} \omega_{\mathrm{r}} \pm \mathrm{j} \omega_{\mathrm{r}} \sqrt{1-\xi_{1}^{2}}$ where, 'p' indicate the real axis pole of the system and $\xi_{1}$ is the damping ratio with passive damping which has been calculated as 0.2 from the Fig 8 .

\section{B. Limitation of Passive Damping}

Passive damping could be helpful in reducing the Q-factor but this is possible up to a certain limit, beyond that passive damping can lead to lower converter efficiency or higher losses. So combination of passive with active damping could be a good practical solution for grid-interactive converter especially medium and high power application.

\section{State-Space BASEd Hybrid PAssive/Active DAMPING}

In this section, the ' $Q$ ' factor (or resonant peak) has been reduced even further by super-imposing suitable control algorithm (active damping) on the passive damping topology. The exact cascaded control loops for controlling resonance [9] would be leading to a large number of nested control loops (here four) as numbers of states are more in this case, which in turn increases the complexity of control structure and especially the complexity tuning the bandwidth of the current controller when switching frequency and resonance frequency are little bit closer to each other as in which case, the bandwidth of the current loop can not be tuned too much for controlling resonance oscillation. Here, state-feedback gain could easily be tuned to control the oscillation without increasing bandwidth of the inner converter current loop as state-feedback loop comes inside the line current controller. Here, the imaginary pole-pair of the passively damped system in Fig 6 are moved even further to the left side of s-plane in order to get better damping (higher $\xi$ and lower 'Q') as shown in Fig. 9, by means of suitable control of PWM converter.

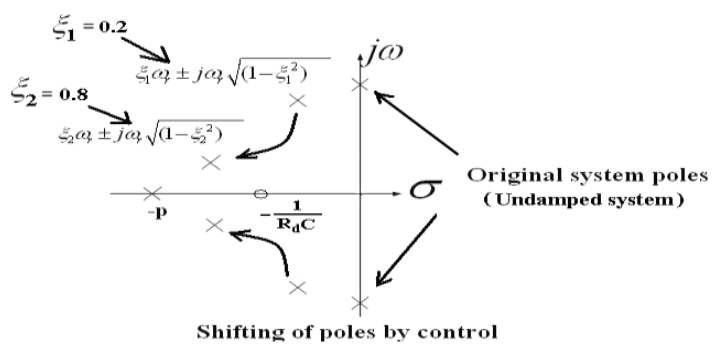

Fig. 9. Movement of imaginary pole-pairs of capacitor voltage $\left(V_{c}\right)$ $V s$ converter output $\left(U_{i n v}\right)$ transfer function by means of state-feedback.

\section{A. Pole-Placement by State-Feedback}

Now, the imaginary pole pairs, $-\xi_{1} \omega_{\mathrm{r}} \pm \mathrm{j} \omega_{\mathrm{r}} \sqrt{1-\xi_{1}^{2}}$ in Fig. 6 are shifted to new pole location $-\xi_{2} \omega_{\mathrm{r}} \pm \mathrm{j} \omega_{\mathrm{r}} \sqrt{1-\xi_{2}^{2}}$ as shown in Fig. 9, where $\xi_{2}>\xi_{1}$, by means of state-feedback control law $\mathrm{U}_{\mathrm{inv}}=-\mathrm{KX}_{\mathrm{H}}$ where $\mathrm{X}_{\mathrm{H}}=\left(\begin{array}{llll}\mathrm{V}_{\mathrm{c}} & \mathrm{V}_{\mathrm{c} 2} & \mathrm{i}_{\mathrm{L} 1} & \mathrm{i}_{\mathrm{L} 2}\end{array}\right)^{\mathrm{T}}$ and ' $\mathrm{K}$ ' is the required state-feedback gain .The new pole positions as shown in Fig. 9, are $\mathrm{s}=0,-\mathrm{p},-\xi_{2} \omega_{\mathrm{r}} \pm \mathrm{j} \omega_{\mathrm{r}} \sqrt{1-\xi_{2}{ }^{2}}$ where $\xi_{2}$ is the new or desired damping ratio, here that is assumed as $0.8\left(\xi_{2}>\xi_{1}\right)$. The state-feedback gain matrix $\mathrm{K}=$ $\left[\begin{array}{lllll}\mathrm{K}_{1} & \mathrm{~K}_{2} & \mathrm{~K}_{3} & \mathrm{~K}_{4}\end{array}\right]$ is calculated so as to shift the pole-pair to desired location.

TABLE I: A TyPiCAl NuMERICAL CALCUlATION OF STATE-FEEDBACK GAIN MATRIX PARAMETERS FOR THE ENTIRE SYSTEM

\begin{tabular}{|l|l|}
\hline \multicolumn{1}{|c|}{$\mathrm{R}_{\mathrm{d}}$} & Gain Matrix parameters for a given \\
& $\mathrm{R}_{\mathrm{d}}$ \\
\hline $25 \Omega$ & $\mathrm{K}_{1}=-1, \mathrm{~K}_{2}=0.6, \mathrm{~K}_{3}=-24.8 \approx-25$, \\
& $\mathrm{K}_{4}=24.8 \approx 25$ \\
\hline
\end{tabular}

It is very clear from the Table I that state-weightages corresponds to the two capacitor voltages $\left(\mathrm{V}_{\mathrm{c} 1}, \mathrm{~V}_{\mathrm{c} 2}\right)$ are negligible compared to state-weightages of two inductor currents $\left(\mathrm{i}_{\mathrm{L} 1}, \mathrm{i}_{\mathrm{L} 2}\right)$ and also weightages of two inductor currents are exactly equal and opposite. It is true for any pole placement and any values of $R_{d}$ as $K_{3}=-K_{4}$. So, in other words, it could be stated that, weightages of inductor currents are higher compared to state weightages of capacitor voltages and it is only required to give proper state-weightage (' $\mathrm{K}$ ') to $\left(\mathrm{i}_{\mathrm{L} 2}-\mathrm{i}_{\mathrm{L} 1}\right)$ to achieve required damping as shown in Fig. 11 where value ' $\mathrm{K}$ ' depends upon the desired damping factor (or desired 'Q'-factor).

\section{B. Improvement of Damping Factor by State-feedback}

The damping ratio is gradually improved from zero (un-damped) to 0.2 (passively damped) and then to 0.8 (hybrid damped) in steps as shown in Fig. 10 by means hybrid damping which is shown in Fig. 10. The gradual or step by step improvement of damping by using hybrid damping for given value of $\mathrm{R}_{\mathrm{d}}(25 \Omega)$ validates the design as well as proposal. The experimental results of the proposed damping are provided to support the theoretical proposal (Fig. 12 to Fig. 14)

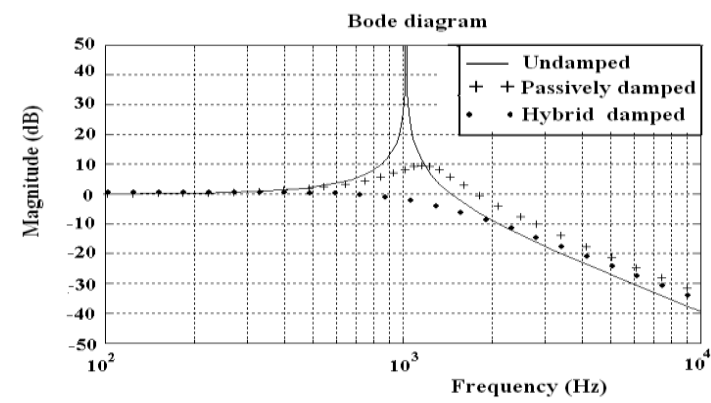

Fig. 10. Gradual reduction of ' $Q$ '-factor $\left(\mathrm{R}_{\mathrm{d}}=25 \Omega\right.$ and $\xi_{1}=0.2$ and $\left.\xi_{2}=0.8\right)$

\section{CONTROL OF PWM CONVERTER FOR EXPERIMENTAL IMPLEMENTATION}

The system in Fig. 1 has been implemented in a lab-setup with 10KVA power converter connected with $415 \mathrm{~V}$ (L-L), $50 \mathrm{~Hz}$ grid. The concept of hybrid damping is verified fully under different DC-bus voltages (Fig. 12 to Fig. 14). The 
entire control structure of front-end converter is divided into three parts like, 1. DC-bus control, 2. Line current control and 3. Inner-most active damping loop. The DC-bus control and line current control are done through conventional PI-controller $[7,8]$ but the active damping has been carried out by means of state-feedback control law as described earlier sections. The grid- synchronization is done with the help of PLL algorithm. The line voltage space vector is made aligned with the ' $q$ '-axis while implementing the PLL algorithm. So, the 'q'-axis becomes active power reference axis and' $d$ '-axis becomes reactive power reference axis in this implementation as can be seen in Fig. 11.

\section{A. Model for Control Design}

The resonance $\left(\mathrm{f}_{0}\right)$ is around $1 \mathrm{KHz}$. Rotating reference frame (vector control) approach is followed for control of DC-link voltage and line side current in order to get better dynamic response and accurate reference tracking. The space vector notation is used.

The power converter is modeled as a gain $\mathrm{G}$ with a first order delay $\mathrm{T}_{\mathrm{d}}(=0.5 \mathrm{Ts})$ where $\mathrm{Ts}$ is the sampling time. So, $\mathrm{G}_{\mathrm{PWM}}=\frac{G}{1+s T_{d}}$. Modeling the LCL-filter in a dq-reference frame gives following equations:

$$
\begin{aligned}
& L_{1} \frac{d i_{L 1}{ }^{d q}}{d t}=V_{c}^{d q}-U_{i n v}^{d q}-j \omega L_{1} i_{L 1}{ }^{d q} \\
& L_{2} \frac{d i_{L 2}{ }^{d q}}{d t}=U_{g}^{d q}-V_{c}^{d q}-j \omega L_{2} i_{L 2}{ }^{d q} \\
& C \frac{d V_{c}^{d q}}{d t}=i_{L 2}^{d q}-i_{L 1}^{d q}
\end{aligned}
$$

Neglecting losses of the converter and of the filter, the power balance equation between grid side and DC-side could be written as

$$
V_{d c} i_{d c}=\frac{3}{2} U_{g q} i_{L 2 q}
$$

The DC-link voltage dynamics can be written as,

$$
C_{d c} \frac{d V_{d c}}{d t}=\frac{3}{2} \frac{U_{g q} i_{L 2 q}}{V_{d c}}-i_{\text {Load }}
$$

\section{B. DC-Link Voltage Control}

The design of the DC-link voltage PI controller parameters $\left(K_{d c}, T_{d c}\right)$ assumes that the delay caused by inner line current loop is around four-sample delay $\left(T_{\text {inner }}=4 T_{s}\right)$ [8]. Assuming that the DC-bus voltage is always closes its reference $V_{d c}{ }^{*}$, the PI controller can be designed by symmetrical optimum method, which is generally used to design the speed controller in dc-drive.

$$
\begin{gathered}
K_{d c}=\frac{2}{3} \frac{V_{d c}{ }^{*} C_{d c}}{a_{d c} T_{i n n e r} U_{g q}} \\
\mathrm{~T}_{\mathrm{dc}}=\mathrm{a}_{\mathrm{dc}}{ }^{2} \mathrm{~T}_{\text {inner }}
\end{gathered}
$$

\section{Decoupling between $d q$ and Line Current Control}

The only disadvantage of this type of control is the coupling between $\mathrm{d}$-axis and q-axis quantities. The decoupling between $\mathrm{d}$ - and q-axis as shown in Fig. 11 helps in properly controlling the DC-quantities.

$$
\begin{gathered}
L_{T} \frac{d i_{L 2 q}}{d t}+U_{i n v q}+\omega L_{T} i_{L 2 d}=U_{g q} \\
L_{T} \frac{d i_{L 2 d}}{d t}+U_{i n v d}-\omega L_{T} i_{L 2 q}=0
\end{gathered}
$$

\begin{tabular}{|c|c|c|}
\hline & Symbols & Values \\
\hline $\begin{array}{l}\text { Filter } \\
\text { arameters }\end{array}$ & $\mathrm{L}_{1}, \mathrm{~L}_{2}, \mathrm{C}_{\mathrm{T}}, \mathrm{R}_{\mathrm{d}}$ & $3 \mathrm{mH}, 3 \mathrm{mH}, 16 \mu \mathrm{F}, 25 \Omega$ \\
\hline $\begin{array}{l}\text { Oontroller } \\
\text { arameters }\end{array}$ & $\mathrm{f}_{0}, \mathrm{Ts}, \mathrm{K}_{\mathrm{dc}}, \mathrm{T}_{\mathrm{dc}}, \mathrm{K}_{\mathrm{p}}, \mathrm{T}_{\mathrm{c}}$ & $\begin{array}{c}1 \mathrm{KHz}, 100 \mu \mathrm{s}, 4.75 \\
1.8 \mathrm{~ms}, 60,450 \mu \mathrm{s}\end{array}$ \\
\hline
\end{tabular}

The couplings have been avoided by adding suitable feed-forward terms ( $U_{\text {invdff, }} U_{\text {invaff }}$ ) to the output of d- and $\mathrm{q}$-axis current controller before giving modulation signal to power converter as in Fig. 11. The line current PI-controller is designed by symmetric optimum method [8].

$$
K_{p}=\frac{L_{T}}{2 T_{c}}, T_{c}=a_{c}^{2} T_{s}
$$

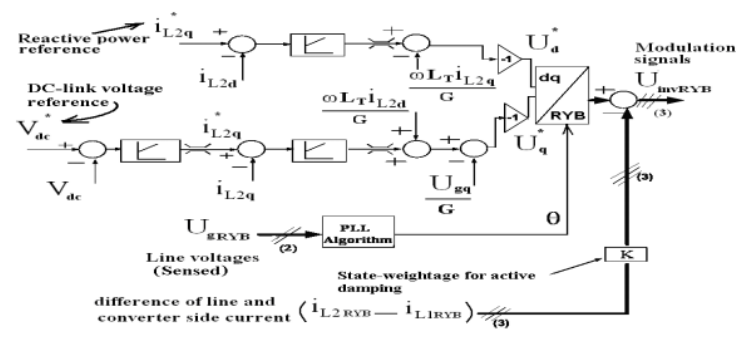

Fig. 11. Complete control structure in grid interactive mode including active damping loop.

\section{EXPERIMENTAL RESULTS}

The experiment has been carried out in laboratory with 10KVA PWM power converter with 415V line-line voltage. Power circuit is shown in Fig 1. The range of DC-bus is in between $600 \mathrm{~V}$ to $700 \mathrm{~V}$ for testing of the DC-bus control. The testing has been done at different operating conditions. The readings are presented at $700 \mathrm{~V}$ DC-link condition as the resonance oscillation is higher at higher DC-bus voltages

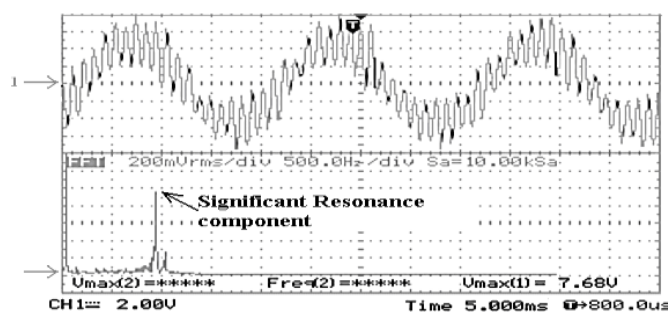

Fig. 12. Measured line-side current with un-damped LCL filter (scale $10 \mathrm{~A} / \mathrm{div})$

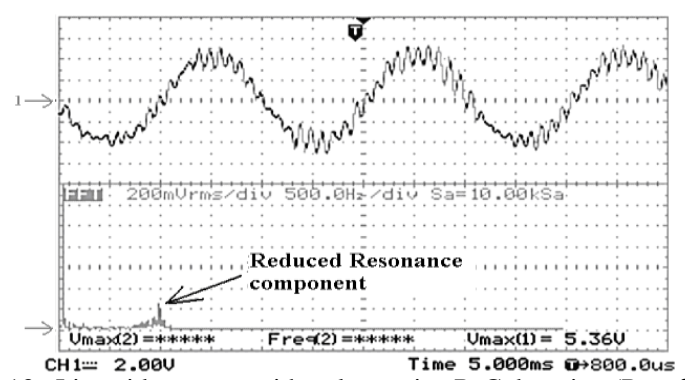

Fig. 13. Line-side current with only passive R-C damping $\left(R_{d}=25 \Omega\right)$ (scale 10A/div). 


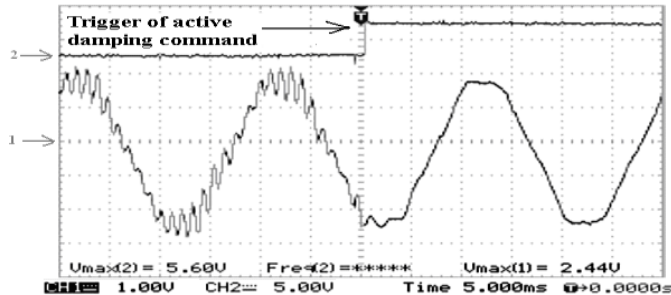

Fig. 14. Line side current with hybrid damping $\left(R_{d}=25 \Omega\right)$ scale (10A/div).

\section{CONCLUSION}

In this paper hybrid approach to damping of LCL filter for active front-end converter is analyzed and fully experimentally verified. It has been found that $\mathrm{R}-\mathrm{C}$ parallel damping in Fig 2 is superior in performance especially in medium and higher power levels. The results of proposed method of hybrid-damping have been verified in frequency domain as well as in time domain.

\section{REFERENCES}

[1] F. Blaabjerg, R. Teodorescu, M. Liserre, and A. V. Timbus, "Over view of control and grid synchronization for distributed powe generation system," IEEE Trans, Ind. Electron, vol. 53, no. 5, pp 1398-1409.

[2] J. R. Rodriguez, J. W. Dixxon, J. R. Espinoza, J. Pontt, and P. Lezana, "PWM regenerative rectifier : State of art," IEEE Trans. Ind. Electron, vol. 52, no. 1 , pp. 5-22, 2005

[3] J. M. Carrasco, L. G. Franquelo, J. T. Bialasiewicz, E. Galvan, R. C. PortilloGuisado, M. A. M. Prats, J. I. Leon, and N. M. Alfonso "Power Elctronic systems for the grid integration of renewable energy sources: A survey," IEEE Trans. Ind. Electron, vol. 53, no. 4, pp. 1002-1016 2006.
[4] IEEE recommended practices and requirements for harmonic control in electrical power systems, IEEE Standard 519, 1992.

[5] IEEE standard for interconnecting distributed resources with electric power systems, IEEE Standard 1547, 2003.

[6] T. C. Y. Wang, Z. Ye, G. Sinha, and X. Yuan, "Output filter design for a grid connected three -phase inverter," GE Global Research Center 2003 report.

[7] M. H. Bierhoff, and F. W. Fuchs, "Active damping for three phase PWM rectifier with higher order line side filters," IEEE Trans. Electron, vol. 56, no. 2, pp. 371-379, 2009.

[8] V. Blasko and V. Kaura "A novel control to actively damp resonance in input LC filter of a three phase voltage source converter," IEEE Trans. Industry Applications, vol. 33, no. 2, pp. 542-550, 1997.

[9] E. Twining and D. G. Holmes, "Grid current regulation of a three phase voltage source inverter with an LCL input filter" IEEE Trans. Power Electron, vol. 18 , no. 3, pp. 888-895, 2003.

[10] M. P. Kazmierkowski and H. Tunia, Auomatic control of power converter fed drives. Amsterdam, The Netherlands: Elsevier, 1994.

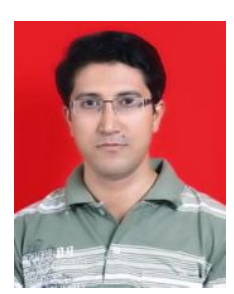

Nilanjan Mukherjee received BEng degree in Electrical Engineering from West Bengal University of Technology, India in 2007. He obtained MEng degree from Indian Institute of Science (IISc), Bangalore, India, in 2009 in the field of Power Electronics where he worked with control of smart grid interactive power converter for distributed generation application. He is now working towards $\mathrm{PhD}$ degree with Power Engineering and Power Electronics group in Aston University, in UK.

He had obtained two years of industrial experience from 2009 to 2011 working in $\mathrm{R} \& \mathrm{D}$ division of Tata Motors Ltd where he was responsible for designing optimized power converter and its control for hybrid energy storage in low power electric vehicle.

His research interest includes control of multilevel converter for smart power system, reliable and intelligent converter topologies for integrating distributed generation to grid and energy storage system.

Mr. Mukherjee is a member of The Institution of Engineering and Technology (IET) in UK. 